\title{
Intervention-point principle of meshless method
}

\author{
YANG JianJun ${ }^{*} \&$ ZHENG JianLong \\ School of Traffic and Transportation Engineering, Changsha University of Science and Technology, Changsha 410004, China
}

Received February 12, 2012; accepted May 4, 2012; published online September 9, 2012

\begin{abstract}
Meshless method is a type of promising numerical approach. But for the method, the convergence is still lack of common theoretical explanations, and the technique of numerical implementation also remains to be improved. It is worth noting that a kind of uniformly defined intervention point is used in many existing schemes. Therefore, the intervention-point principle is proposed. The viewpoint is likely to give a reasonable explanation for the inaccuracy and instability of the collocation method. Based on the principle, a design process for a new scheme was demonstrated. Some initial numerical tests were also offered. The results have revealed the intervention point to take effect on convergence, suggested a construction concept using intervention point for meshless collocation method, and presented a new scheme of meshless method for application.
\end{abstract}

meshless method, intervention point, convergence, collocation method, approximation

Citation: $\quad$ Yang J J, Zheng J L. Intervention-point principle of meshless method. Chin Sci Bull, 2013, 58: 478-485, doi: 10.1007/s11434-012-5471-x

Nowadays, mechanics must fully take advantage of modern computational technology, to solve any macroscopic and practical problems of science and technology, and computational method is very important [1]. Some conventional computational methods have been widely applied to solve practical and complicated problems, such as finite element method (FEM), finite difference method (FDM) and boundary element method (BEM). These methods are all based on meshes. Meshless method is a unique computational method, which gives solution using a set of arbitrarily distributed nodes. This kind of properties breaking away from meshes can bring some benefits for the method. One is of great convenience to execute, for setting up nodes is much easier than constructing meshes. Another is the flexibility for application. The method can effectively eliminate the connatural drawbacks of using meshes, and is suitable for handling some challenging problems, such as large deformation problem, structure decomposition problem, adaptive analysis problem, fluid dynamics problem, and explosion and impact problem. Moreover, for some objects difficult to describe by meshes, meshless method also has advantages, such as simulating galaxies on cosmic scale in celestial

*Corresponding author (email: yangiianjun01@126.com) mechanics, portraying polyatomic crystals in microphysics, imitating cells or molecules in biomechanics. Therefore, meshless method with its ingenious tactics and great promise has become the most active research subject in recent decades [2-5].

In China, the research into meshless methods has also become very lively. Some impressive contributions have been reported and applied, such as the development on the radial basis functions (RBF) by $\mathrm{Wu}$ [6], the least square collocation meshless method (LSC) by Zhang et al. [7], the hybrid boundary node method (HBNM) by Zhang et al. [8], the boundary knot method (BKM) and the singular boundary method (SBM) by Chen et al. $[9,10]$, the complex variable moving least-square approximation method (CVMLS) by Cheng et al. [11], the application research on MLPG method by Long et al. [12], the Galerkin boundary node method (GBN) by Li et al. [13], and so on.

However, the time has not come for cheering. For a powerful meshless method, many obstacles still remain to be solved. We have not developed a uniform theoretical perspective on convergence of the method, or to guarantee accuracy and stability. At present, dozens of schemes for the method have been proposed, and new ones are still emerging. For those successful schemes, we believe that 
their success must have the essence of a general consistency. If we stay on the comparison and differentiation for specific methods, we will only see the complicated phenomenon. That is not helpful for us to discover the uniform theory for the method and to develop more competitive schemes.

\section{What is the intervention point?}

Based on the derivation rule of system equations, or discretization method, we can roughly classify meshless methods into some basic types, shown in Table 1 [2-4].

The collocation method is initially used in the early study of meshless method [14,15], and typically uses only field nodes to build discrete system equations. The method is very simple and direct, and perfectly fit for the spirit of scattered nodes discretization of meshless method. Unfortunately, the method is usually instable and inaccurate for solving. Therefore, the method had been somewhat restricted in its application.

The least square method is a kind of discretization approach based on least square weighted residuals method. The method usually uses a kind of "auxiliary point" to build system equations [7]. Compared to the collocation method, solving by the least square method is obvious improvement in stability and accuracy.

All the weak form methods, III, IV and V in Table 1, need a kind of shadow mesh, although this shadow mesh may be global, local or border. In addition, these methods need to rely on "integral point" to evaluate integrals. The weak form methods really have advantages of excellent stability and satisfactory accuracy, and bring prosperity for the research of meshless method [16-18].

Moreover, a special collocation method has not escaped our attention. It is the double grid diffuse collocation method (DGDC) [19]. In the method, a kind of so-called "evaluation point" was used. For the performance of the method, the authors stated that "exhibit good convergence properties".

Following upper remarks, it is not difficult to find a very interesting phenomenon: meshless method using some special "point", which is different from field node, have varying degrees of improvements in accuracy and stability. Yet this kind of point is the absence for ordinary collocation method. For the point, we can give a uniform definition: when we build the discrete equations for meshless method, a kind of point may be used, which is different from field node or not for domain discretization; then we define it as the "intervention point".

\section{Intervention-point principle}

First, we will put forward a proposition: intervention point is an important condition to ensure convergence for meshless method. We name this proposition as the intervention-point principle. In particular, we will discuss the proposition on the precondition of using moving least square (MLS) approximation method in this paper. Then our topic will be locked in the subject of discretization method, so as to avoid interference of approximation method. If we scrutinize the effectiveness of the intervention point in meshless method, its functionality can be summarized into three main aspects.

(1) Increasing accuracy and consistency of approximation. This is a universal and essential functionality of the intervention point. Meshless method is based on the weighted residual method. For a random target node $\boldsymbol{x}_{l}$, when its field variable $u_{I}(\boldsymbol{x})$ is approximated by an approximation function $\tilde{u}_{I}(\boldsymbol{x})$, the residual will be produced, which is denoted as $R_{I}(\tilde{u}) \neq 0$. Obviously,

$$
\delta=\left\|\tilde{u}_{I}^{(d)}(\boldsymbol{x})-u_{I}^{(d)}(\boldsymbol{x})\right\|, \quad \lim _{\delta \rightarrow 0} R_{I}\left(\tilde{u}^{(d)}\right) \rightarrow 0 .
$$

Table 1 A subtotal of meshless methods

\begin{tabular}{|c|c|}
\hline Discretization methods & Specific schemes \\
\hline I Collocation method & $\begin{array}{l}\text { Smooth particle hydrodynamics method (SPH), free mesh difference method (FMD), vortex method, finite point } \\
\text { method (FPM), hp-cloud method, double grid diffuse collocation method (DGDC) }\end{array}$ \\
\hline III Galerkin method & $\begin{array}{l}\text { Diffuse element method (DEM), element free Galerkin method (EFG), reproducing kernel particle method (RKPM), } \\
\text { partition of unity finite element methods (PUM), meshfree point interpolation method (MPIM), meshfree radial-basis } \\
\text { point interpolation method (MRPIM) }\end{array}$ \\
\hline IV Petrov-Galerkin method & $\begin{array}{l}\text { The meshless local Petrov-Galerkin method (MLPG), local point interpolation method (LPIM), local radial-basis } \\
\text { point interpolation method (LRPIM) }\end{array}$ \\
\hline V Boundary method & $\begin{array}{l}\text { Boundary node method (BNM), boundary particle method (BPM), local boundary integral equation method (LBIE), } \\
\text { hybrid boundary node method (HBNM), boundary knot method (BKM), boundary cloud method (BCM), boundary } \\
\text { point interpolation method (BPIM), boundary radial-basis point interpolation method (BRPIM), singular boundary } \\
\text { method (SBM), Galerkin boundary node method (GBN) }\end{array}$ \\
\hline VI Combinatorial method & $\begin{array}{l}\text { Meshfree weak-strong form method (MWS), meshless Galerkin least square method (MGLS), hybrid mesh-free finite } \\
\text { volume difference method (HMFVDM) }\end{array}$ \\
\hline
\end{tabular}


It is explained that the convergence of meshless method depends on the accuracy of approximation. In addition, approximation is different from interpolation, and we should not only pay attention to the approximation accuracy of the target node, but also think about the accuracy of information collection from other field nodes (supported nodes). This is the requirement of approximation consistency for the numerical method. We should realize that a direct approximation approach (Figure 1(a)) is a deliberately balanced but not complete strategy. This kind of approximation strategy is used in ordinary collocation method, and practice shows that the strategy is not effective enough. An improved scheme is an indirect approximation strategy (Figure 1(b)), in which, target node gets field information from intervention point, but not from field node directly. All the weak form methods have used this strategy, although field information transfer from intervention point to target node may be not by approximation (so we used a dashed line to denote this kind of difference in the figure). Yet, intervention point participating in approximation is a certainty. Also note that DGDC has used the indirect approximation strategy, and the process from intervention point to target node is an approximation relation. Another improved scheme is a compound approximation strategy (Figure 1(c)). Same, intervention point also joins approximation, but the field information of intervention point will be attached to target node, and a special solving technique is needed. The least square method has used the strategy. Indeed, for a certain method, intervention point is not only for approximation. But the contribution for intervention point to increase approximation accuracy and consistency is worthy of recognition.

(2) Constructing discretization method. For some discretization methods, intervention point is essential. For example, weak form methods need integral point, and least square method maybe need auxiliary point. These discretization methods created intervention point, say it in another way, it is the intervention point to support those discretization methods. Actually, some discretization method with good convergence could also be given a reasonable explanation in the approximation sense. For example, all the weak form methods from III, IV and V categories in Table 1, can avoid executing a higher order derivative approximation according to the governing equation. That is very helpful for

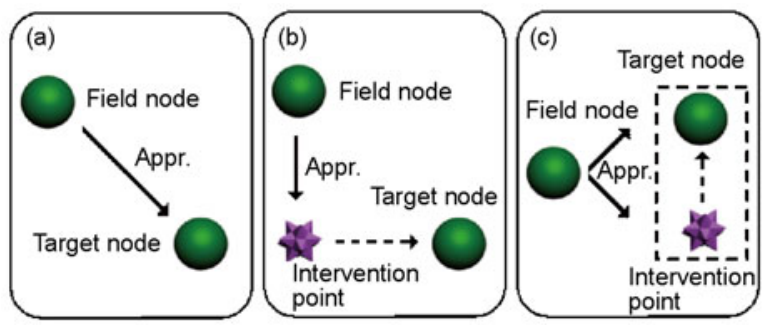

Figure 1 Schematics of approximation strategy in meshless method. (a) Direct approximation (node to node); (b) indirect approximation (node to intervention point to node); (c) compound approximation (node to node, plus node to intervention point). the methods showing fast convergence.

(3) Strengthening stability. For meshless method, stability can be expressed by two aspects: numerical solution is insensitive for arbitrary field variable, and for relatively arbitrary field nodes distribution. This is essentially a performance of approximation stability. We can get some messages from existing methods: appropriate density of intervention points is helpful for stability. For example, both of EFG and MLPG have shown better stability. We should notice that integral points are densely used in the methods. But their stability could not be ensured, when we choice less integral points.

These introductions are essentially a general description of the effect on convergence for intervention point. In fact, for a specific method, intervention point not only plays a certain function, more likely, plays a combination of multiple functions. The intervention-point principle may give a simple and uniform construction concept for meshless method: intervention point is needed beyond field node. Except for the collocation method, intervention point is indeed necessary for other discretization methods. But for the collocation method, this may be a breakthrough viewpoint, and may also be a controversial one.

The principle also hints at a possibility: we could set up a uniform convergence theory based on intervention point for meshless method. This kind of theoretical support is surely needed for the method. All the discretization schemes of meshless method are based on the weighted residual method, and the residuals come from approximation. Research has shown that all weighted residual methods are uniformly convergent [20]. But it is undeniable that different discretization method probably gives a different performance for solving. Even for those methods similarly based on the collocation method, using intervention point or not, will show very different results. These give us an important revelation: for meshless method, the uniform convergence of the weighted residual method is conditional, and intervention point is an important role.

The principle is also helpful to break some stereotypes. On the road of collocation method, a "stop" sign has probably been set up. Judging with experience, the collocation method is really underwhelmed by its performance. However, explained by the intervention-point principle, the weakness of the collocation method is not natural but short of intervention point. Indeed, we cannot hope the method to make bricks without straw. Based on the principle, some new collocation methods will possibly be developed. Next, we will demonstrate a scheme design process, which can also be taken as a support and annotate for the interventionpoint principle.

\section{A scheme design example}

According to the intervention-point principle, meshless 
method need intervention point, and intervention point should serve approximation. Following this train of thought, we will design a new scheme based on collocation discretization method. We name this scheme as the meshless intervention point method (MIP).

For meshless method, to construct meshless approximation is a primary task. MLS is generally considered to be one of the best schemes with a reasonable accuracy [16-18]. So we will choose MLS [21] for approximation function in MIP, and its functional image is shown in Figure 2. It is well known that the accuracy of the approximation is very important for the performance of a meshless method. However, we cannot place our whole hope on the approximation function to execute satisfactory approximation unconditionally; it is a hard mission to complete for the function in most cases. In our concept of scheme design, we need to create conditions for accurate and stable approximation, especially for derivative approximation. The intervention point can supply favorable conditions to attain the target.

The approximation function not only carries out approximation of field information, but also takes a role of field information transmitter. In approximation process, a reasonable small size of supported domain, namely locality, is the base of approximation accuracy for the calculation point (target point). Only if the supported domain is small enough, a random local field function cut off by it can be a simple one; the approximation function with limited ability can probably give an accurate approximation. In field information transmitting process, a reasonable big size of supported domain, namely coverability, is the base of effectual transmitting. Only if the supported domain is big enough, more supported points (field node) will take part in the action of field information transmitting, the link relation between the calculation point and the supported points can be stable. This need of locality and coverability is a contrary request of both big and small for the supported domain size. That is so called "supported domain paradox". Using intervention point, we can get off the hooks. Next, we will elaborate our designing ideas for MIP.

(1) Locking the scale of local domain. By the mediation

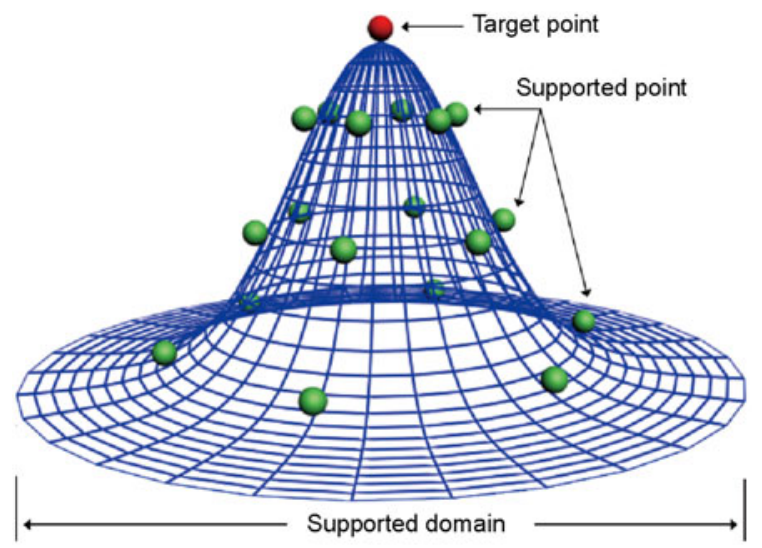

Figure 2 MLS approximation function. of intervention point, we can separate a supported domain $\Omega_{\mathrm{S}}$ into two domains with different functions (Figure 3 ), the small one $\Omega_{\mathrm{L}}$ named as the local domain is in charge of accurate approximation, and the big one $\Omega_{\mathrm{C}}$ named as the cover domain is in charge of information transmitting. Thus, the size of local domain can be defined freely and restricted effectually. The approximation function is usually needed to implement derivative approximation, and derivative operator can enlarge the approximation errors. Since approximation on the local domain is more reasonable, then derivative approximation on the local domain is certainly more accurate. So we should put the derivative approximation process on the local domain usually.

(2) Creating conditions for higher degree basis. This is closely related with the effect of intervention point to lock the scale of local domain. MLS approximation usually uses a polynomial basis to construct the trial/approximation function, only if the degree of basis is high enough, approximation capacity of the trial function can be powerful enough, and therefore approximation can be accurate enough. If no intervention point participates in, for a given field nodes distribution scheme, a higher degree basis means more terms produced, which need more field nodes to play as supported points, then the size of supported domain will be expanded accordingly, and the potential field function on a bigger domain will be more complicated. In other words, when we upgrade the trial function, the approximation target will grow to be more complicated. For approximation accuracy, the trial function just like a hamster in a rotating cage, no matter how it try its best to run, the delicious cookie is always far from touch. Therefore, in order to avoid the approximation accuracy out of control, 2-degree basis is usually used. But, the lower degree of basis may be not enough, especially for derivative approximation. When intervention point is used, local domain can be locked, and intervention point can be used more freely, and higher degree basis can be used more flexibly on local domain. Thus, the accuracy of derivative approximation can be guaranteed for the target node $\boldsymbol{x}_{\boldsymbol{I}}$.

(3) Ensuring the consistency of approximation. For the

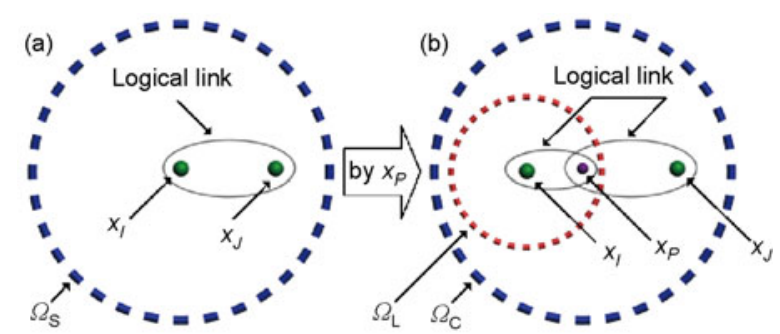

Figure 3 Supported domain separating by intervention point. (a) The simple supported domain $\Omega_{\mathrm{S}}$, field node $\boldsymbol{x}_{J}$ is a support for target node $\boldsymbol{x}_{I}$; this kind of logical link of approximation is a simple chain structure. (b) Here $\boldsymbol{x}_{P}$ is the intervention point, former $\Omega_{\mathrm{S}}$ is separated out of two domains, local domain $\Omega_{\mathrm{L}}$ and cover domain $\Omega_{\mathrm{C}}$; for approximation, $\boldsymbol{x}_{P}$ is a support for $\boldsymbol{x}_{I}$, and $\boldsymbol{x}_{J}$ is a support for $\boldsymbol{x}_{P}$; the logical link between $\boldsymbol{x}_{I}$ and $\boldsymbol{x}_{J}$ is turned into a hinge type structure. 
accurate solution of meshless method, only an accurate approximation for the target node $\boldsymbol{x}_{I}$ is not enough. We should also ensure the accuracy of field information collection from the supported node $\boldsymbol{x}_{J}$. We call this kind of requirement the consistency of approximation. It would be like two horses drawing a wagon together; faster one with slow one will not be fast. Using the intervention point $\boldsymbol{x}_{P}$, we could let $\boldsymbol{x}_{P}$ close to or even get to $\boldsymbol{x}_{J}$, and then we could capture accurate filed information from $\boldsymbol{x}_{J}$. Therefore, the consistency of approximation could be ensured. Also, we should realize that a smaller scale $\Omega_{\mathrm{L}}$ is favorable for $\boldsymbol{x}_{I}$, but may be unfavorable for $\boldsymbol{x}_{J}$. Hence, we should reasonably set the local domain size taking care of each other concerns.

Based on these approximation tactics using intervention point, MIP's basic approximation formulas are written as

$$
\begin{gathered}
\tilde{u}_{I}(\boldsymbol{x})=\sum_{p=1}^{N_{p}} \phi_{p}(\boldsymbol{x}) \hat{u}_{p} \text { on } \Omega_{\mathrm{L}}, \\
\hat{u}_{p} \cong \tilde{u}_{p}(\boldsymbol{x})=\sum_{J=1}^{N_{J}} \phi_{J}(\boldsymbol{x}) \hat{u}_{J} \text { on } \Omega_{\mathrm{C}},
\end{gathered}
$$

where $\hat{u}_{*} \equiv u_{*}(\boldsymbol{x})=u\left(\boldsymbol{x}_{*}\right), \phi_{*}(\boldsymbol{x})$ denotes the MLS shape function corresponding to the point $\boldsymbol{x}_{*}, N_{p}, N_{J}$ denote the number of intervention points $\boldsymbol{x}_{P}$ in $\Omega_{\mathrm{L}}$ and field nodes $\boldsymbol{x}_{J}$ in $\Omega_{\mathrm{C}}$, respectively. We can eliminate $\hat{u}_{p}$ by substituting eq.

(3) into eq. (2), and obtain

$$
\tilde{u}_{I}(\boldsymbol{x})=\sum_{p=1}^{N_{p}} \sum_{J=1}^{N_{J}} \phi_{p}(\boldsymbol{x}) \phi_{J}(\boldsymbol{x}) \hat{u}_{J} .
$$

Commonly, its $d$-th order derivative approximation formula can be written as

$$
\tilde{u}_{I}^{(d)}(\boldsymbol{x})=\sum_{p=1}^{N_{p}} \sum_{J=1}^{N_{J}} \phi_{p}^{(d)}(\boldsymbol{x}) \phi_{J}(\boldsymbol{x}) \hat{u}_{J} .
$$

In order to demonstrate the numerical implementation for MIP method, we consider a linear Poisson's equation in a global domain $\Omega$ bounded by $\Gamma$. The problem is defined as

$$
\left\{\begin{array}{cc}
\nabla^{2} u(\boldsymbol{x})=f(\boldsymbol{x}), & \boldsymbol{x} \in \Omega, \\
u(\boldsymbol{x})=\bar{u}(\boldsymbol{x}), & \boldsymbol{x} \in \Gamma_{u}, \\
\partial u(\boldsymbol{x}) / \partial n=\bar{t}(\boldsymbol{x}), & \boldsymbol{x} \in \Gamma_{t},
\end{array}\right.
$$



Figure 4 Schematics of meshless intervention point method (MIP). where $u(x)$ is the field function, $f(\boldsymbol{x})$ is the given distributing function (body load) on the problem domain, $\bar{u}(\boldsymbol{x})$ is the known displacement function on the essential boundary $\Gamma_{u}$, $\bar{t}(\boldsymbol{x})$ is the given load function on the natural boundary $\Gamma_{t}$, and $n$ is the outer normal vector of $\Gamma_{t}$. The domain discretization of MIP method is shown in Figure 4. The problem's numerical system equation can be written as

$$
\boldsymbol{K} \cdot \boldsymbol{U}=\boldsymbol{F},
$$

where $\boldsymbol{K}$ is the global stiffness matrix, $\boldsymbol{U}$ is the global displacement vector which need to be solved, and $\boldsymbol{F}$ is the global load vector. Then, the MIP discrete equations for an arbitrary calculation node $\boldsymbol{x}_{I}$ are given as

$$
\begin{gathered}
\boldsymbol{K}_{I J}= \begin{cases}\sum_{p=1}^{N_{p}} \sum_{J=1}^{N_{J}} \phi_{p, i i}(\boldsymbol{x}) \phi_{J}(\boldsymbol{x}), & \boldsymbol{x}_{I} \in \Omega, \\
\sum_{p=1}^{N_{p}} \sum_{J=1}^{N_{J}} \phi_{p}(\boldsymbol{x}) \phi_{J}(\boldsymbol{x}), & \boldsymbol{x}_{I} \in \Gamma_{u}, \\
\sum_{p=1}^{N_{p}} \sum_{J=1}^{N_{J}} \phi_{p, n}(\boldsymbol{x}) \phi_{J}(\boldsymbol{x}), & \boldsymbol{x}_{I} \in \Gamma_{t},\end{cases} \\
\boldsymbol{F}_{I}= \begin{cases}f_{I}(\boldsymbol{x}), & \boldsymbol{x}_{I} \in \Omega, \\
\bar{u}_{I}(\boldsymbol{x}), & \boldsymbol{x}_{I} \in \Gamma_{u}, \\
\bar{t}_{I}(\boldsymbol{x}), & \boldsymbol{x}_{I} \in \Gamma_{t},\end{cases}
\end{gathered}
$$

where $(\cdot)_{, i i}$ denotes $\partial^{2}(\cdot) / \partial i^{2}$, and $i$ is a dimensional component of $\boldsymbol{x}$, and $(\cdot)_{, n}$ denotes $\partial(\cdot) / \partial n$. It might also be noted that the approximation tactic of MIP is focused on struggling with the challenge of derivative approximation. Therefore, the approach is not strictly necessary for dealing with the essential boundary condition. In most of the cases, we can use the common collocation method for $\boldsymbol{x}_{I} \in \Gamma_{u}$ to simplify the calculation, which is defined as

$$
\boldsymbol{K}_{I J}=\sum_{J=1}^{N_{J}} \phi_{J}(\boldsymbol{x}), \quad \boldsymbol{x}_{I} \in \Gamma_{u}
$$

Since intervention points in $\Omega_{\mathrm{L}}$ are used locally and temporarily, we can use a standard mould creating the $\Omega_{\mathrm{L}}$ with intervention points for a target node $\boldsymbol{x}_{I}$. Furthermore, the intervention-point mould can be designed specially to achieve the balance of accuracy and efficiency.

\section{Numerical examples}

\subsection{An infinite plate with a circular hole problem}

Firstly, we consider a classic example, the infinite plate with a circular hole [17], which is forced a tensile load $\left(q_{x= \pm \infty}=1\right)$ in $x$ direction. Due to symmetry, only the upper right quadrant of the plate with finite structure is modeled, shown in Figure 5(a). We solved the problem with materials parameters of $E=10^{3}$ and $v=0.3$. For the problem, the numerical solutions are shown in Figure 5(b)-(d). It can be seen that the present method can give satisfactory solution 

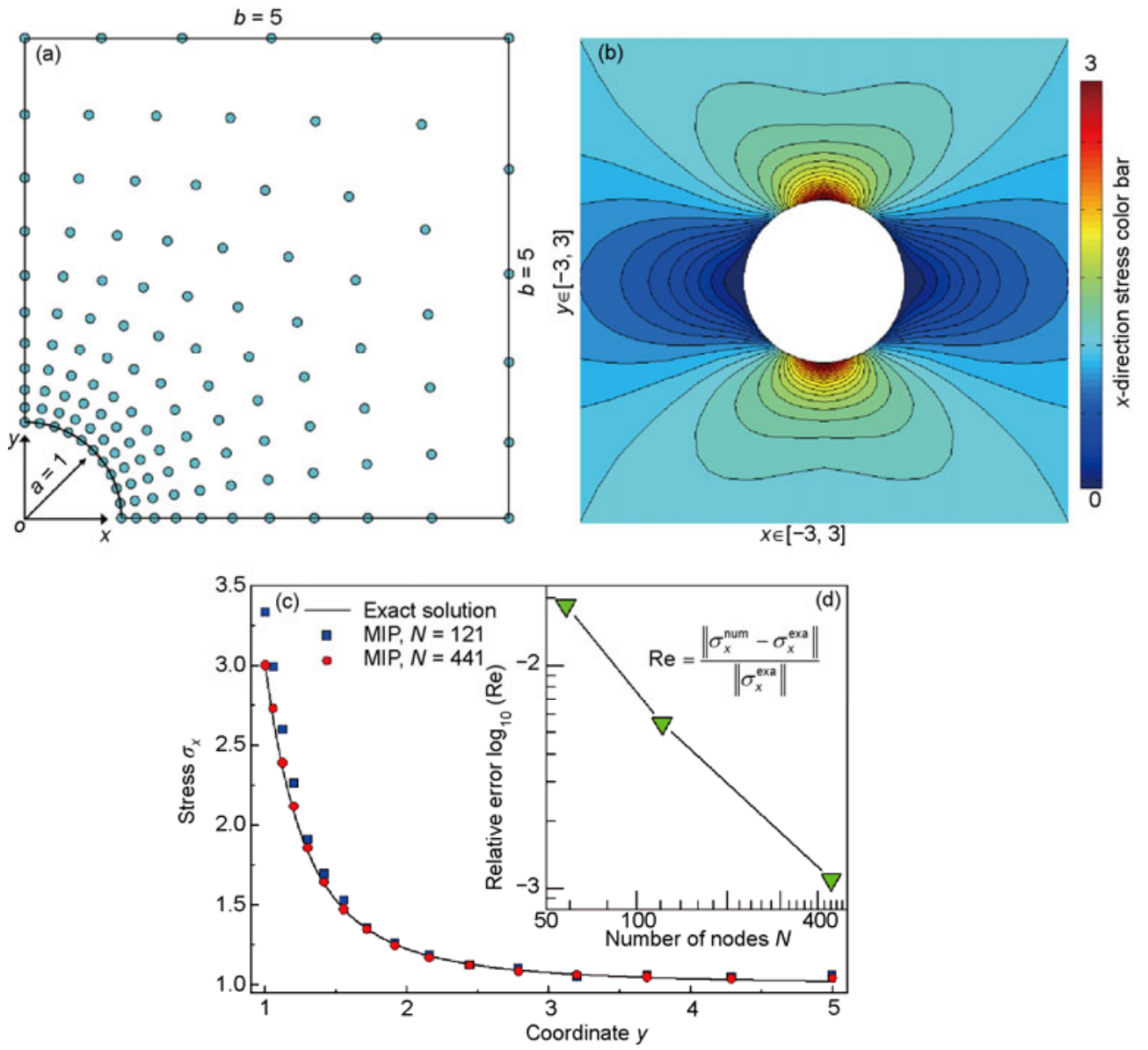

Figure 5 The infinite plate with a circular hole problem. (a) The discrete model of the problem; (b) the distribution of $x$-direction stress around the hole; (c) comparison of numerical solutions and exact solutions for $x$-direction stress at $x=0$; (d) the relative errors for numerical solution.

and shows good convergence for the problem.

\subsection{A transistor heat conduction problem}

MIP can also be easily applied to heat conduction problem. Consider a transistor steady-state heat conduction problem, shown in Figure 6(a). The inner (MESHLESS) and outer boundaries are both given temperature conditions. We solve the problem with $H=4, L=25$, inner boundary condition $t_{0}=42$, and outer boundary condition $T_{0}=95$. We get the numerical solution of the problem with the isothermal chart distribution in the medium of the transistor, which is shown in Figure 6(b). With the problem, we can also test the present method which can be easily applied to the problems with complicated geometric boundary.

\subsection{An atomic chain forced vibration problem}

Meshless method can easily cope with some particle structure problems. Consider a liner atomic chain system building by $N=101$ atoms, which is fixed at one end, and forced by impact load at another, as shown in Figure 7(a) and (b). We solve the problem with atoms space $d=0.1$, then $L=100 d$, (a)
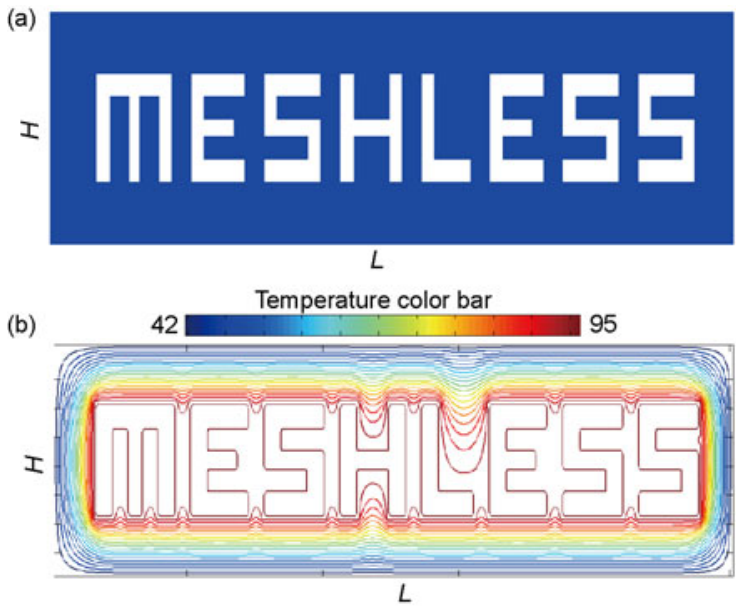

Figure 6 The transistor heat conduction problem. (a) Geometric model of the problem; (b) isothermal chart in medium of the transistor.

elastic constant of atomic bonding force $E=10^{4}$, the mass of an atom $m=L / N$ (unit). We used the Newmark method to solve the dynamical problem, and took time step as $\Delta t=5$ $10^{-5}$, and then we can get the solution as shown in Figure 7(c) and (d). It can be seen that the present method can also 
(a)
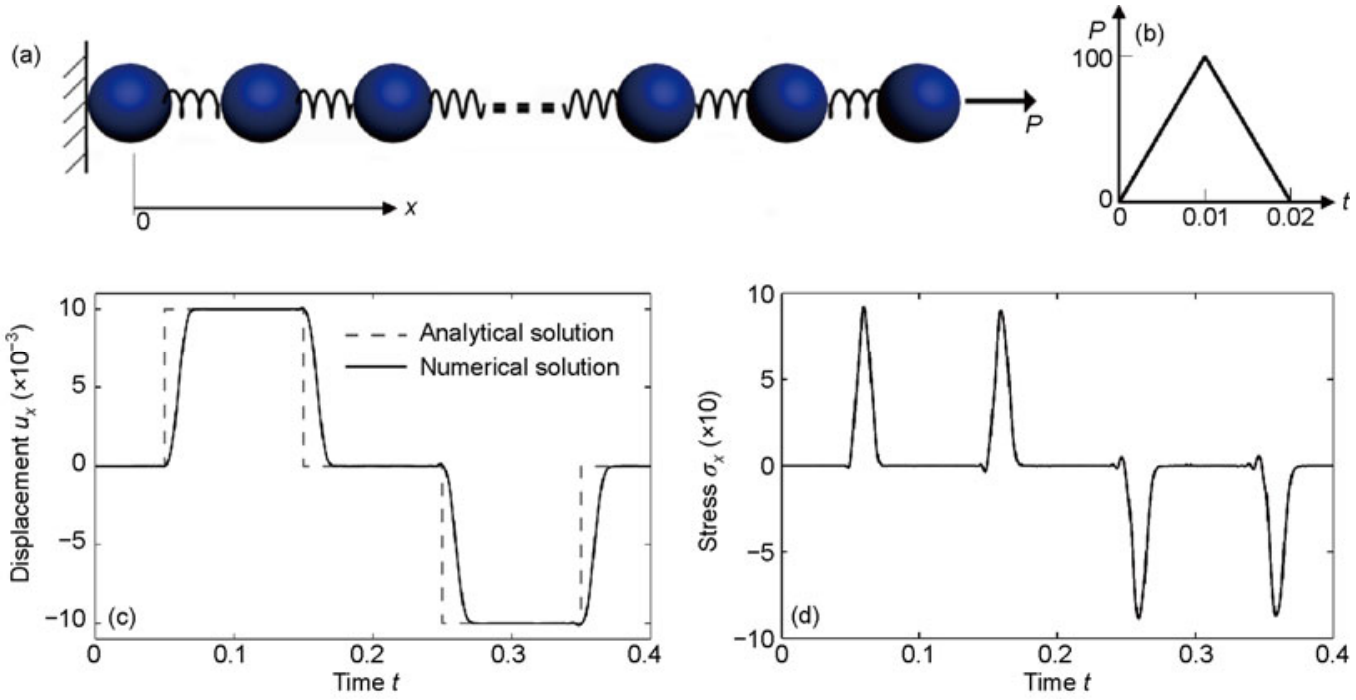

Figure 7 The atomic chain forced vibration problem. (a) The mechanical model; (b) force diagram of outer dynamic load; (c) the displacement wave of the mid atom; (d) the stress wave of the mid atom.

give a satisfactory solution for the problem.

\section{Conclusions and outlook}

In traditional concepts, there is no special position for intervention point in meshless method. But in practice, we have used it in many special schemes casually. The collocation method without intervention point possibly remained a fatal flaw, and this may be the reason for the discretization method making slow progress. The present interventionpoint principle may be to inject new vitality into collocation method, and support meshless method on a way of simplicity and flexibility. The principle also gives an optional view for meshless method to construct a uniform convergence theory, this kind of theoretical support is necessary for a mature and powerful meshless method. It is still questionable for the principle applied to some other approximation methods besides MLS. Overall, we are cautiously optimistic about that. In essence, the principle is proposed as a new opinion, though it is crude and nascent, it could be helpful to inspire the research.

In order to test and explain the principle, we have proposed a new scheme, the meshless intervention point method (MIP). We feel that MIP has some potential advantages. MIP is a simple pure meshless method, also with satisfactory accuracy, stability and flexibility. Observant readers may have noticed that the discretization mode of MIP is somewhat similar to that of MLPG. It indicates that reasonable methods are similar in essence. This kind of similarity can be explained successfully by the intervention-point principle, but cannot by the particular discretization method. Inspired by this, EFG is also a successful scheme, can we use the principle to eliminate the shadow meshes of the method, and convert it into a kind of promising collocation method? Using the principle, can we improve some existing methods? Can we develop some new valuable methods? Anything is possible, as long as our mankind stay thinking.

We thank Zhou Z G, Li Y Y, Qian G P and Guan H X for discussion. This work was supported by the National Natural Science Foundation of China (51038002), the Planned Science and Technology Project of Hunan Province, China (2010FJ6023) and the Open Fund of Key Laboratory of Road Structure and Material of Ministry of Transport (Changsha, kfj100201).

1 Zhong W X, Cheng G D. Trans-century computation mechanics in China (in Chinese). In: Zhou G Z, ed. Scientific and Technological Progress and Discipline Development. Beijing: China Science and Technology Press, 1998. 24-28

2 Belytschko T, Krongauz Y, Organ D, et al. Meshless method: An overview and recent developments. Comput Methods Appl Mech Eng, 1996, 139: 3-47

3 Gu Y T, Ding H. Recent developments of meshless method (in Chinese). Adv Mech, 2005, 35: 323-337

4 Zhang X, Liu Y, Ma S. Meshfree methods and their applications (in Chinese). Adv Mech, 2009, 39: 1-36

5 Atluri S N, Shen S. The basis of meshless domain discretization: The meshless local Petrov-Galerkin (MLPG) method. Adv Comput Math, 2005, 23: 73-93

$6 \mathrm{Wu}$ Z M. Multivariate compactly supported positive definite radial functions. Adv Comput Math, 1995, 4: 283-292

7 Zhang X, Liu X H, Song K Z, et al. Least square collocation meshless method. Int J Numer Methods Eng, 2001, 51: 1089-1100

8 Zhang $\mathbf{J}$ M, Yao Z H, Li H. A hybrid boundary node method. Int J Numer Methods Eng, 2002, 53: 751-763

9 Chen W, Tanaka M. A meshless, exponential convergence, integration-free, and boundary-only RBF technique. Comput Math Appl, 2002, 43: 379-391

10 Chen W, Fu Z J. A novel numerical method for infinite domain potential problems. Chin Sci Bull, 2010, 55: 1598-1603

11 Cheng Y M, Peng M J, Li J H. The complex variable moving least-square approximation and its application (in Chinese). Chin J Theor Appl Mech, 2005, 37: 719-723

12 Long S Y. A local Petrov-Galerkin method for the elasticity problem (in Chinese). Acta Mech Sin, 2001, 33: 508-517

13 Li X L, Zhu J L. Galerkin boundary node method for exterior Neu- 
mann problems. J Comput Math, 2011, 29: 243-260

14 Lucy L. A numerical approach to testing the fission hypothesis. Astron J, 1977, 82: 1013-1024

15 Gingold R A, Moraghan J J. Smoothed particle hydrodynamics: Theory and applications to non-spherical stars. Mon Not Roy Astron Soc, 1977, 18: 375-389

16 Nayroles B, Touzot G, Villon P. Generalizing the finite element method: Diffuse approximation and diffuse elements. Comput Mech, 1992, 10: 307-318

17 Belytschko T, Lu Y Y, Gu L. Element free Galerkin methods. Int J
Numer Methods Eng, 1994, 37: 229-256

18 Atluri S N, Zhu T. A new meshless local Petrov-Galerkin (MLPG) approach in computational mechanics. Comput Mech, 1998, 22: 117-127

19 Breitkopf P, Touzot G, Villon P. Double grid diffuse collocation method. Comput Mech, 2000, 25: 199-206

20 Qiu J B. Theory and Application of Weighted Residuals Method (in Chinese). Beijing: China Astronautic Publishing House, 1991

21 Yang J J, Zheng J L. Stability of moving least squares approximation (in Chinese). Acta Math Appl Sin, 2012, 35: 637-648

Open Access This article is distributed under the terms of the Creative Commons Attribution License which permits any use, distribution, and reproduction in any medium, provided the original author(s) and source are credited. 